\title{
Itinéraires Itinéraires
}

Littérature, textes, cultures

2010-2 | 2010

Les blogs

\section{Blog, fragment et altérité}

\section{Caroline Angé}

\section{OpenEdition}

\section{Journals}

Édition électronique

URL : http://journals.openedition.org/itineraires/2050

DOI : 10.4000/itineraires.2050

ISSN : 2427-920X

Éditeur

Pléiade

Édition imprimée

Date de publication : 1 juillet 2010

Pagination : 141-146

ISBN : 978-2-296-12012-9

ISSN : $2100-1340$

Référence électronique

Caroline Angé, « Blog, fragment et altérité », Itinéraires [En ligne], 2010-2 | 2010, mis en ligne le 01 juillet 2010, consulté le 23 avril 2019. URL : http://journals.openedition.org/itineraires/2050 ; DOI : 10.4000/ itineraires.2050

\section{(c) (1) $\Theta$}

Itinéraires est mis à disposition selon les termes de la licence Creative Commons Attribution - Pas d'Utilisation Commerciale - Pas de Modification 4.0 International. 


\title{
Blog, fragment et altérité
}

\begin{abstract}
Among the several writing practices, there are fragments about blogs. However, there is a need to distinguish the fragmentation coming from softwares and those from the writing practices themselves. Some authors write their immediate thoughts as well as reflections about themselves. Those writings have only the appearance of fragments. Bringing these two practices together shows the importance of the reader who writes comments in blogs.
\end{abstract}

Keywords : weblog, readers, diaries, fragment, technology

Mots clés : blogs, lecteur, écriture de soi, fragment, médiations

Dans l'histoire des dispositifs d'écriture, il est des médiations qui tendent à reconfigurer ce qui lie un genre, une pratique, un message, un support. Le blog, dispositif de publication en ligne, tisse et hybride des formes discursives, linguistiques et énonciatives qu'il fait tenir ensemble tout en les revisitant. Ce faisant, ce dispositif est travaillé par une interrogation sur les genres, eux-mêmes renouvelés par des écritures et des pratiques différenciées. Nous nous attacherons ici à envisager le blog sous la focale de la forme texte ${ }^{1}$ fragment afin de mettre l'accent sur la dimension communicationnelle dont est porteur ce rapprochement. La perspective ici convoquée permettra de reconnaitre des analogies formelles entre d'un côté l'écriture fragmentaire et de l'autre certaines formes textuelles du blog. Pour ce faire, nous nous appuierons sur les résultats de notre recherche sur les modalités de construction du sens liées à l'écriture fragmentaire du texte à l'hypertexte ${ }^{2}$, et plus précisément sur une hypothèse que nous expliciterons : le fragment comme

1. Le fragment est ici entendu comme « forme texte » afin de souligner l'interdépendance entre la manière de dire (le contexte de l'énonciation) et ce qui est dit. C'est en portant une attention toute particulière à l'étude du mode d'apparaître du fragment (son unité formelle et spatiale) qu'on appréhendera l'écriture fragmentaire.

2. Caroline Angé, La Question du sens. Écrire et lire le fragment. Du texte à l'hypertexte, thèse de doctorat de $3^{\mathrm{e}}$ cycle, sciences de l'information et de la communication, Université Paris 13 - MSH Paris Nord, 2005. 
forme impose un certain rapport au lecteur, une relation singulière, en provoquant un type d'adresse au lecteur du point de vue de l'énonciation et de la spatialité de $1^{\text {'objet textuel }}{ }^{3}$. Deux questions guideront ces éléments de réflexion : en quel sens les pratiques d'écriture sur le blog ont-elles à voir avec le fragment? En quoi cette filiation générique est-elle éclairante?

\section{L'essence relationnelle d'une forme}

Afin de considérer la portée heuristique du fragment dans l'étude du dispositif de publication en ligne hétérogène qu'est le blog, il convient de dire quelques mots du périmètre notionnel lié à cette forme. Que signifie écrire et lire en fragments?

Si le fragment est une figure marquante, une présence qui n'a pas attendu notre époque pour se mouvoir dans différentes représentations symboliques, l'histoire nous montre que c'est une forme complexe sur le plan lexical et terminologique. Si l'on considère le fragment dans sa forme même, on distinguera le fragment inachevé temporaire (destiné à être complété par le lecteur), le fragment morcelé (reflet chaotique du monde), le fragment romantique qui accède à la dignité de l'œuvre, le fragment effet accidentel d'une altération « ce qui subsiste » d'un univers textuel supposé (mythe de l'un ou du tout), le fragment production spontanée et concentrée de l'esprit (ce qui résulte du choix de l'auteur ou de l'éditeur). Ces déterminations témoignent d'une forme peu compatible avec une définition préalable et stable dont rendraient compte des critères structurels ou des paramètres de longueurs. Cela tient à la multiplicité des formes dans lesquelles l'écriture fragmentaire s'est incarnée, qui ne peuvent se réduire à une préoccupation technique ou méthodologique mais plutôt à une posture intentionnelle. Nous l'envisageons ici comme forme texte c'est-à-dire comme réalité signifiante prise dans une structure communicationnelle d'écriture-lecture. Sans entrer dans la diversité des causes et la disparité de formes relative aux contextes historiques, intellectuels et sociaux de cette forme, le fragment donne à lire et à voir. En d'autres termes, il provoque d'une manière singulière « l'autre », le lecteur.

Une approche sémiotique permet d'en donner quelques propriétés qui nous mettent sur la voie de la situation de communication de cet objet textuel et de la manière dont il fait sens. Tout d'abord, d'un point de vue morphologique, le mode « d'apparaître » de la forme, comme détachée d'une autre à laquelle elle renvoie, nous introduit à l'interaction de ce qui se joue entre le texte (le fragment), le sujet (écrivant) et autrui (lecteur supposé). Comme le montre Henri Quéré : - il y a un sujet écrivant qui s'approprie un objet textuel, ce qui indique « un rapport cognitif modalisé selon le savoir ou le croire $[\ldots] »$;

- il y a une « brisure diversement imprimée et ouverte » qui affirme sémiotiquement une « disjonction » suspendue et en attente;

3. Caroline Angé, « Le fragment comme forme texte : à propos de Fragments d'un discours amoureux », Communication \& Langages, $\mathrm{n}^{\circ} 152$, juin 2007, p. 23-34. 
- il y a une façon d'être, d'apparaître entre le détaché et le rattaché;

- il y a la suggestion "d'une soudure à faire [...], comme institution d'un programme du faire, qu'il s'agisse pragmatiquement de conjoindre ou de ressouder $[\ldots] \gg$ des morceaux textuels ${ }^{4}$.

De cette analyse, il ressort une spécificité communicationnelle qui tient à la manière dont la forme se donne à lire. L'entre deux visuellement dit l'Autre, le convoque, l'interpelle. Le fragment informe son lecteur d'un contenu tout en lui faisant savoir par là même que quelque chose n'est pas. Le fragment présuppose de l'implicite. On touche là au contrat de lecture du fragment et à sa dimension opératoire. L'écriture de pensées détachées engage, dans ses procédures de sens, l'autre qui viendrait cohabiter et coopérer. La stratégie de l'objet textuel fragment engage ce lecteur supposé à qui l'on suggère de lier, d'élaborer ces liens qui ne sont pas. Certes, on s'adresse toujours à quelqu'un, mais la manière dont le fragment comme forme s'offre au regard de son lecteur instaure une relation singulière dans le rapport à l'autre.

\section{Distinctions médiatiques}

\section{La morphologie d'une forme éditoriale}

Il est question d'éclatement, de dispersions, de parcellaire dans les discours sur notre contemporanéité sans toujours souligner la complexité de ce que recouvre la problématique du fragment.

Le fragment est célébré d'une manière toute particulière dans l'appréhension du numérique à tel point qu'évoquant une interface de blog, on parle de « règne du fragment ${ }^{5}$ » pour définir les transformations subies par l'écrit dans les processus d'inscription et de gestion du texte d'un outil de publication. On trouve là une première distinction qui mérite d'être introduite quant à la structure matérielle du texte, à son substrat technique autrement dit à ce qui tient au support. Les dispositifs techniques opèrent à partir de fragments et notamment lorsqu'il s'agit d'extraction de données. La fragmentation résulte alors des médiations liées au dispositif. Cela n'est pas spécifique au blog mais concerne les modalités techniques de mises en ligne de contenu à des degrés de granularité différents et ce, quels que soient les outils utilisés. Cette première strate aboutit aux conditions pratiques et matérielles qui président à l'existence du fragment. Ce que le philosophe Alain Renaud-Alain comprend comme « la dissolution de toute solidité ${ }^{6} »$ dans les couches d'écritures

4. Henri Quéré, Intermittences du sens : études sémiotiques, Paris, PUF, 1992, p. 75-76.

5. Valérie Jeanne-Perrier, "Des outils d'écriture aux pouvoirs exorbitants? », Réseaux, $\mathrm{n}^{\circ} 137,2006 / 3$, p. 126.

6. Alain Renaud-Alain, «La multiplicité numérique, de l'éclatement à la dissipation», dans Jean-Pierre Mourey (dir.), Logiques de fragmentation, Saint-Étienne, Publication de l'université de Saint-Étienne, 1996, p. 255. 
induites par les effets programmatiques. L'opérativité logico-technique produit une fragmentation qui se perpétue dans l'éclatement de l'écran.

Ainsi, par le tissage des énoncés, les interfaces des blogs se caractérisent par une fragmentation inhérente à la logique structurelle de l'outil. Les blocs de textes, la gestion des blancs et des marges configurent un espace fragmenté aussi bien d'un point de vue morphologique que structurel dans la mesure où les envois successifs présupposent « une énonciation fragmentée et datée ${ }^{7}$ ».

\section{Le sens d'une forme textuelle}

En premier lieu, la disposition fragmentée des énoncés sur la page écran tient davantage au dispositif de publication «blog » qu'à la forme texte fragment. Il convient alors de s'attacher plus précisément à « l'en-soi » des billets publiés afin de voir s'ils réfèrent aux modèles originaires du genre.

Dans la limite étroite du corpus ${ }^{8}$ pris en exemple, on trouve dans les segments de phrases choisis une filiation à l'écriture fragmentaire avec des morceaux de textes qui s'affirment comme autosuffisants. L'expérience de l'écriture dont rendent compte ces énoncés est associée à une liberté, dans la pensée propre au genre, par laquelle s'affirme un regard singulier porté par la subjectivité d'une énonciation. C'est la quête, l'élaboration progressive que l'on retrouve dans la publication de soi. Surgissements de l'élan, de l'instant, de la volonté attestent d'une indépendance du propos dans l'écriture de soi. Les tâtonnements, les ébauches montrent un je qui se peint par touches. La pensée se prend elle-même pour objet, l'enjeu est la présence à soi et écrire est un moyen de cette réflexion. La pensée se critique, se réinterprète, décèle ses présupposés et sa logique. Il s'agit alors de repérer les démarches d'inflexion de la pensée sur sa propre activité. La discontinuité s'impose pour dire l'instant. Le blog Fragments ${ }^{9}$ témoigne de cette forme d'écriture fragmentaire proche du journal intime. Un langage formel de « vous à moi » tend à exprimer des états de conscience ou les pensées du moment. Des épigraphes (citations mises en exergue), des titres tels « fragment premier, naissance de la conscience » ou « fragment noir » et extraits de textes poétiques encadrent parfois le propos voire s'y substituent. Dès lors, les fragments sont tissés et mis en abîme dans d'autres formes textuelles.

\section{Le blog ou l'apparence d'une forme?}

Plus encore, la filiation avec le genre se trouve dans la poétique du blog avec une conception de la connaissance qui revendique la liberté spirituelle

7. Oriane Deseilligny, « Du journal intime au blog », Communication \& Langages, $\mathrm{n}^{\circ} 150$, mars 2008, p. 52.

8. Les blogs pris en exemple ici appartiennent à un corpus de blogs hétéroclites ayant pour trait commun un rapport étroit à la forme fragment (revendiqué comme pratique d'écriture, comme contenu ou thème de réflexion) mais ne relèvent pas en propre d'un genre reconnu de la blogosphère (intime, journalistique, etc.).

9. http://fragments.over-blog.fr/30-index.html, consulté le 6 juin 2008, désormais inaccessible. Le pseudonyme « $\mathrm{K} »$ renvoie à l'auteur. 
du fragment. Qu'il s'agisse de prendre sa pensée pour objet ou des sujets d'ordres divers, la forme exprime la relativité de ce qui est dit. L'aléatoire, l'émotion et le contact sont de mise dans certains blogs qui dans leur matière et dans leur forme témoignent d'un esprit mobile en quelque sorte en état de poésie. Les blogs de la catégorie « sagesse » hébergés par « over-blog.com » - tels que le blog du pseudonyme « Jahman ${ }^{10}$ » intitulé Fragments de pensées et résidus - se situent dans ce style personnel délibéré qui s'affranchit d'un mode d'exposition systématique. Cette matière textuelle qui s'annonce comme du fragment est composée d'articles constituant des entrées successives sur les thèmes de la connaissance, de la spiritualité, de la philosophie. Le « je » est en quelque sorte « source de vérité » pour atteindre l'absolu. La méthode solipsiste est assumée comme socle de l'argumentation. La vérité n'est réelle, vraie que « pour moi en présence de moi-même ». Les énumérations concourent à faire des énoncés affectifs la voie vers la connaissance. Pour autant, tout en s'appuyant sur la rhétorique du fragment, ces énoncés n'ont que l'apparence $d u$ genre et deviennent le prétexte à l'expression d'une subjectivité, sans atteindre la rigueur conceptuelle de l'énoncé propre au fragment.

\section{L'autre, le lecteur dans le texte}

La forme texte fragment comme pratique d'écriture réflexive est une pratique poétique qui, tout à la fois, dit et montre ce qu'elle dit. Le lecteur de fragments comme tout autre lecteur participe de la révélation du sens. Plus encore, l'absence de liens formels entre les morceaux de texte pose une implication singulière du lecteur. L'alternance entre les énoncés fragmentaires et les interstices postule une ouverture et un manque. Là réside l'essence même de l'acte de lecture de fragments, à savoir le fait d'avoir donné sens par l'acte de liaison. Acte de lecture qui met en scène d'une manière singulière l'écart irréductible, la distance entre « je » et « l'autre ». Certains blogs montrent une segmentation visuelle au travers de différents blocs qui scindent le texte avec des citations et autres éléments paratextuels. Ce faisant, ils inscrivent sémiotiquement le lecteur dans les interstices. Mais, on touche là aux limites de la filiation avec le genre.

L'énonciation dans le blog fait de l'acte de lecture un élément constitutif du dispositif qui acquiert une réalité effective par les formes de communication mises en œuvre par l'auteur/lecteur. L'échange communicationnel triangulé associe toujours un tiers, le public-lecteur comme troisième personne (lui, ils, eux) qui « valide » l'écriture. Un billet se visite, se lit, se commente. Dès lors, le blanc se donne à lire comme un espace dont les commentaires proposent des formes de remplissage. Le lecteur n'est plus seulement inscrit sémiotiquement dans les interstices mais comme instance énonciative. La valeur communicationnelle de la forme texte fragment se trouve alors exacerbée dans une équivalence productive énonciateur/lecteur.

10. http://sagesses.over-blog.com/archive-08-2006.html, consulté le 14 avril 2010. 
L'extrait du blog de Pierre Assouline ${ }^{11}$, livrant sa critique de l'ouvrage d'Éric Marty sur Fragments d'un discours amoureux, donne à voir des commentaires qui se succèdent, expriment une diversité des points de vue, des méta-commentaires qui vont de la citation à de tout autres considérations subjectives. Ces traces de lecture montrent que les spécificités relationnelles des formes d'énonciations du blog vont bien au-delà de la situation de communication supposée par la forme texte fragment.

\section{De l'hybridité formelle du blog}

Si des traces du genre « fragment » sont repérables dans la blogosphère tout en s'entremêlant à d'autres genres, ces pratiques revêtent l'apparence de cette « forme texte » plus qu'elles ne l'expriment.

Ces quelques éléments de réflexion permettent de conclure sur la portée heuristique des filiations et notamment du fragment pour éclairer la spécificité de ce dispositif de publication. Cela permet de souligner l'importance des opérations techniques et intellectuelles qui donnent une forme aux textes donnés à lire. Il y a bien une fragmentation du texte d'écran dans les formats d'expression des blogs qui a une incidence sur les conditions d'interprétation et de manipulation des textes. Plus encore, il y a dans le fragment l'institution d'un programme du faire qui invite le lecteur à en combler l'incomplétude. La filiation avec les formes textuelles du blog invite à s'attacher aux traces de lectures comme définissant la poétique du genre. La forme des fragments sur ce dispositif médiatique conduit à questionner l'autre figure : le lecteur supposé. Certains lecteurs se proposent de rendre compte des liens qu'ils tissent entre les fragments en livrant leurs commentaires, d'autres se contenteront de visiter. Ainsi, la filiation générique bute sur la question de l'altérité et la distance maintenue entre soi et l'autre quoiqu'en disent les discours de promotion sur les blogs. L'écart entre soi et l'autre subsiste nécessairement, et ce même si le lecteur propose sa lecture des fragments.

Scruter le fragment dans le blog rend compte des médiations de la communication, de leur complexité dans une société soumise au double processus d'hypermédiatisation et d'hypersonnalisation de ses échanges.

Caroline Angé

Université Stendhal - Grenoble 3 - GRESEC

11. La référence au blog de Pierre Assouline porte sur les commentaires de sa lecture critique de l'ouvrage d'Éric Marty et non sur le blog en lui-même qui ne présente pas de corrélations avec le genre « fragment ». Voir http://passouline.blog.lemonde.fr/2006/05/29/2006_05_fragments_dun_d/\#comments, consulté le 14 avril 2010. Il s'agit ici simplement de rendre compte de la présence d'un lecteur-écrivant propre à la dynamique interactionnelle du blog. 